Cleide Mara Mazzotti de

OLIVERA FRANZIN ${ }^{1}$

JoÃo LuIZ PINTO e SIIIVA ${ }^{2}$

Belmiro Gonçalves Pereira ${ }^{1}$

SérGio TADEu MARTINS MARBA'

SirLe SICENI MORAIS ${ }^{3}$

TAtiana AMARAL ${ }^{4}$

ERICK BARBOSA RESZKA ${ }^{4}$

\section{Centralização de fluxo sanguíneo fetal: comparação entre os índices diagnósticos}

\author{
Brain-sparing effect: comparison of diagnostic indices
}

Artigo original

Palavras-chave

Fluxo sanguíneo fetal Ultrassonografia doppler Ultrassonografia pré-natal Circulação placentária Resultados da gravidez Artérias umbilicais Artéria cerebral média/ultrassonografia

Keywords

Fetal blood Ultrasonography, doppler Ultrasonography, prenatal

Placental circulation

Pregnancy outcome

Umbilical arterries

Middle cerebral artery/ultrasonography

\section{Resumo}

OBJETIVO: descrever os resultados perinatais adversos em pacientes com centralização de fluxo sanguíneo fetal, utilizando a relação entre os índices de pulsatilidade das artérias cerebral média e umbilical (IPACM/IPAU) e entre os índices de resistência das artérias cerebral média e umbilical (IRACM/IRAU), assim como comparar os dois índices diagnósticos. MÉTODOS: foram incluídas 151 gestantes com diagnóstico de centralização de fluxo sanguíneo atendidas na maternidade da Universidade Estadual de Campinas, cujo parto ocorreu até 15 dias após o diagnóstico ultrassonográfico. Foram considerados como resultados perinatais adversos: índice de Apgar inferior a sete no quinto minuto, internação em UTI neonatal, concepto pequeno para a idade gestacional, sofrimento fetal agudo, mortalidade perinatal, hipoglicemia, policitemia, enterocolite necrosante, hemorragia cerebral, hemorragia pulmonar, anemia, septicemia, doença de membrana hialina, síndromes convulsivas, síndrome de hiper-reflexia e insuficiência renal. As frequências dos resultados perinatais adversos (RPA) para as relações cérebro-placentária foram comparadas utilizando-se o teste exato de Fisher ou o do $\chi^{2}$ de Pearson, considerando-se como estatisticamente significativo 0 nível de 5\%. Os resultados perinatais adversos foram avaliados de acordo com a idade gestacional, utilizando-se o teste de tendência de Cochrane-Armitage. RESULTADOS: os resultados perinatais adversos para o grupo em que os dois índices apresentaram-se alterados constatou que 62,5\% dos recém-natos necessitaram de internação em UTI, conceptos pequenos para a idade gestacional (PIG) ocorreram em 75,2\%, sofrimento fetal agudo em $35,3 \%$, hipoglicemia em 84,4\%, policitemia em 8,3\%, enterocolite necrosante em 4,2\% e hemorragia cerebral em 2,1\%. Constatou-se associação significativa das relações IPACM/IPAU e IRACM/IRAU, no decorrer da idade gestacional, para necessidade de cuidados intensivos neonatais, fetos pequenos para a idade gestacional, septicemia, enterocolite necrosante, insuficiência renal, doença de membrana hialina e anemia. Não houve diferença significativa entre um ou outro índice para resultados perinatais adversos.

\section{Abstract}

PURPOSE: to describe adverse perinatal outcomes in patients with fetal blood flow centralization, using the relationship between the pulsatility indexes of the middle cerebral and umbilical arteries (MCAPI/UAPI), and between the resistance indexes of the middle cerebral and umbilical arteries (MCARI/UARI), as well as to compare both diagnostic indexes. METHODS: 151 pregnant women with diagnosis of blood flow centralization, attended to at the maternity hospital of Universidade Estadual de Campinas, whose delivery occurred up to 15 days after the ultrasonographic diagnosis, were included. It was considered as adverse perinatal outcomes: Apgar index lower than 7 at the fifth minute, permanence in neonatal ICU, small fetus for the gestational age, severe fetal suffering, perinatal death, hypoglycemia, polycythemia, necrotizing enterocolitis, brain hemorrhage, lung hemorrhage, anemia, septicemia, hyaline membrane disease, convulsive syndromes, hyperreflexia syndrome and kidney insufficiency. Rates of the perinatal adverse outcomes (PAO) for the brain-placentary ratios have been compared, using Fisher's exact or Pearson's $\chi^{2}$ tests, at $5 \%$ significance level. Adverse perinatal outcomes according to the gestational age have been evaluated using the Cochrane-Armitage test for trend. RESULTS: the adverse perinatal outcomes for the group with the two indexes altered were: $62.5 \%$ of the newborns needed to be placed in an ICU, 75.2\% were small for the gestational age (SGA), 35.3\% were under severe
Correspondência:

Cleide Mara Mazzotti de Oliveira Franzin Cidade Universitária "Zeferino Vaz", $s / n$ Caixa Postal 6181 (EP 13083-970 - Campinas (SP), Brasil E-mail: cleide.franzin@gmail.com

Recebido

25/5/09

Aceito com modificacoẽes
Departamento de Tocoginecologia da Faculdade de Ciências Médicas da Universidade Estadual de Campinas - UNICAMP Campinas (SP), Brasil.

'Professor do Departamento de Tocoginecologia da Faculdade de Ciências Médicas da Universidade Estadual de Campinas UNICAMP - Campinas (SP), Brasil.

2 Professor Titular do Departamento de Tocoginecologia da Faculdade de Ciências Médicas da Universidade Estadual de Campinas - UNICAMP - Campinas (SP), Brasil.

${ }^{3}$ Estatística do Departamento de Tocoginecologia da Faculdade de Ciências Médicas da Universidade Estadual de Campinas UNICAMP - Campinas (SP), Brasil.

${ }^{4}$ Acadêmico do Curso de Medicina da Faculdade de Ciências Médicas da Universidade Estadual de Campinas - UNICAMP Campinas (SP), Brasil. 
fetal suffering, $84.4 \%$ had hypoglycemia, $8.3 \%$ polycythemia, $4.2 \%$ necrotizing enterocolitis, and $2.1 \%$ brain hemorrhage. There has been significant association between the MCAPI/UAPI and MCARI/UARI ratios along the gestational age, and the need for neonatal intensive care, small fetuses for the gestational age, septicemia, necrotizing enterocolitis, kidney insufficiency, hyaline membrane disease, and anemia. There has been no significant difference between the two indexes of adverse perinatal outcome.

\section{Introdução}

Há mais de uma década, constatou-se que o uso rotineiro da doplervelocimetria em gestações de alto risco estava associado à redução da mortalidade perinatal, da frequência de intervenções cirúrgicas fetal, da indução de trabalho de parto e das cesáreas por sofrimento fetal ${ }^{1}$.

Atualmente, estudos randomizados e por meta-análise confirmaram que a utilização da doplervelocimetria da artéria umbilical em fetos com restrição de crescimento resultaram em significativa redução da mortalidade perinatal e de intervenções iatrogênicas ${ }^{2,3}$.

Os fetos com perfusão placentária comprometida apresentam redistribuição de fluxo sanguíneo, com aumento da resistência vascular periférica fetal e redução compensatória da resistência sanguínea no cérebro, efeito denominado "preservação cerebral" ${ }^{4-6}$, conhecido no meio médico como "centralização de fluxo sanguíneo". Esse mecanismo é conhecido de longa data por meio de experimentos em ovelhas?.

A doplervelocimetria da artéria cerebral média, quando alterada, indica diminuição da resistência ao fluxo sanguíneo e está diretamente relacionada à intensidade da hipoxemia; ou seja, há evidências de vasodilatação cerebral em hipoxemia leve a grave e, em casos de hipoxemia extrema, ocorre diminuição da resistência vascular, provavelmente como reflexo do aumento da pressão intracraniana causada pelo edema cerebral ${ }^{8}$. No estágio inicial de redistribuição sanguínea, ocorre aumento da resistência na artéria umbilical e diminuição da resistência nas artérias cerebrais. Portanto, o diagnóstico de centralização de fluxo sanguíneo fetal pode ser aferido por alterações dos índices de impedância das artérias cerebrais ou por meio da relação entre os índices de pulsatilidade das artérias umbilical e cerebral média (IPAU/IPACM) .

Tais estudos foram confirmados com a relação inversa, ou seja, o índice de pulsatilidade da artéria cerebral média dividido pelo índice de pulsatilidade da artéria umbilical (IPACM/IPAU). Os valores inferiores a 1,08 a partir da $30^{a}$ semana de gestação foram considerados anormais, com acurácia diagnóstica de $90 \%$ para resultados perinatais adversos ${ }^{10}$.

A relação entre os índices de resistência da artéria cerebral média e da artéria umbilical (IRACM/IRAU) menor ou igual à unidade também identifica fetos com risco para restrição de crescimento e morbidade neonatal severa $^{11-13}$. Em um estudo, a sensibilidade e especificidade para resultados perinatais adversos com esse índice foi de 64,1 e $72,7 \%$, respectivamente ${ }^{13}$. Posteriormente, outros estudos demonstraram maior sensibilidade e especificidade das relações cérebro-placentárias na identificação de resultados perinatais adversos, quando comparada com a artéria umbilical como único parâmetro ${ }^{14,15}$.

A autorregulação do fluxo sanguíneo cerebral geralmente ocorre como resposta à perfusão placentária deficiente ${ }^{16}$, mas também tem sido descrita como resposta a procedimentos invasivos fetais ${ }^{17}$ e em algumas doenças cardíacas fetais $^{18}$.

O diagnóstico do bem-estar fetal é uma das principais preocupações do obstetra, e embora as relações entre os índices cerebrais e placentários sejam apontadas como uma forma de elucidar esse diagnóstico, poucos estudos com número significativo de casos são relatados na literatura, muitas vezes com intervalo de tempo muito longo entre o exame doplervelocimétrico e o nascimento do concepto. Em alguns casos, quando um índice está normal e o outro alterado, pairam dúvidas quanto à presença ou ausência de centralização de fluxo sanguíneo fetal.

Este estudo tem por objetivo analisar os resultados perinatais adversos associados à centralização de fluxo sanguíneo fetal diagnosticada pelas relações IPACM/ IPAU e IRACM/IRAU, assim como a comparação entre os dois índices.

\section{Métodos}

Foram incluídas neste estudo 151 gestantes com diagnóstico prévio de centralização de fluxo sanguíneo fetal, realizado pelo Serviço de Ultrassonografia do Centro de Atenção Integral à Saúde da Mulher (CAISM) da Universidade Estadual de Campinas (UNICAMP), atendidas entre janeiro de 2002 e dezembro de 2003.

O diagnóstico de centralização de fluxo sanguíneo fetal foi instituído por meio das fórmulas: IPACM/IPAU inferior a $1,08^{10}$ ou IRACM/IRAU inferior à unidade ${ }^{11}$. $\mathrm{O}$ índice de pulsatilidade foi definido como (S - D)/ $/ \mathrm{M}^{19}$ e o índice de resistência foi definido como $(S-D) / S^{20}$, significando: $\mathrm{S}$ a velocidade sistólica máxima da onda espectral, $\mathrm{D}$ a velocidade diastólica final e $\mathrm{M}$ a velocidade média da onda espectral.

Foram excluídas do estudo as gestantes cujos fetos apresentassem malformações, se houvesse dificuldades na 
extração do concepto, prolapso de cordão umbilical, parto ocorrido fora da instituição ou parto ocorrido após 15 dias do último exame ultrassonográfico no qual se confirmou o diagnóstico de centralização de fluxo sanguíneo fetal.

A idade materna variou de 13 a 46 anos, com média de 28,2. As complicações da gravidez mais frequentes foram as síndromes hipertensivas. Apenas uma paciente teve parto assistido com fórceps; 25 evoluíram para parto normal, e a maioria (125 pacientes; $82,7 \%$ ) foi submetida à cesárea.

A doplervelocimetria foi realizada com a gestante em decúbito dorsal, na ausência de movimentos respiratórios fetais. O cursor de amostra de volume foi ajustado a um nível mínimo para evitar interferência com os vasos adjacentes, mantendo-se entre $23 \mathrm{~mm}$. Para remover as interferências de baixas frequências causadas pelo movimento das paredes dos vasos, utilizou-se filtro de 100 $\mathrm{Hz}$. Obteve-se fluxo arterial usando equipamento marca Toshiba, modelo Powervision 6000, com transdutor convexo de 3 a 4,2 $\mathrm{MHz}$. Após boa audição e visibilização dos sinais a imagem foi congelada, realizando-se planigrafia da onda com calíperos eletrônicos. Após ajuste adequado do ângulo entre o cursor e o vaso, as medidas de velocidades sistólica, diastólica e média, assim como os índices de impedância, foram calculados eletronicamente pelo equipamento.

A potência acústica do modo Doppler com mapeamento em cores se manteve abaixo dos padrões estabelecidos pela Food and Drug Administration (FDA) em 1976, e pela International Federation of Gynecology and Obstetrics $(\mathrm{FIGO})^{21}$ para a ultrassonografia fetal, com intensidade média do pico espacial temporal de $57 \mathrm{~mW} / \mathrm{cm}^{2}$.

Após visualização da artéria umbilical com mapeamento em cores, colocou-se o cursor no lúmen do vaso que se apresentava paralelo à onda ultrassônica, com ângulo de incidência inferior a 30 graus. Foram realizadas medidas em alça livre, longe da inserção placentária e fetal $^{22}$. Para avaliação da artéria cerebral média, obtevese corte transversal do polo cefálico, incluindo o tálamo e o cavum do septo pelúcido (plano em que o diâmetro biparietal é medido). A seguir, movimentando o transdutor caudalmente, no plano paralelo ao previamente tomado, identificou-se a asa maior do osso esfenoide entre as fossas média e anterior ${ }^{5}$. Usou-se o mapeamento em cores para identificar o polígono de Willis, e a artéria cerebral média foi facilmente identificada como o maior ramo anterolateral do polígono, dirigindo-se à borda lateral da órbita. Posicionou-se o cursor no lúmen da artéria cerebral média próxima ao transdutor, no trecho compreendido entre um centímetro da sua origem na artéria carótida interna. $\mathrm{O}$ ângulo entre o cursor e o vaso não excedeu a 30 graus. Os exames foram realizados pelos médicos do Setor de Ultrassonografia do CAISM.
O infarto placentário foi definido como área de necrose isquêmica ocasionada por redução do suprimento sanguíneo. O diagnóstico foi emitido pelo Departamento de Anatomia Patológica da Faculdade de Ciências Médicas da UNICAMP.

Consideramos como resultados perinatais adversos: índice de Apgar inferior a sete no quinto minuto, internação em Unidade de Terapia Intensiva Neonatal, concepto pequeno para a idade gestacional (PIG), sofrimento fetal agudo, mortalidade perinatal, hipoglicemia, policitemia, enterocolite necrosante, hemorragia cerebral, hemorragia pulmonar, anemia, septicemia, doença de membrana hialina, síndromes convulsivas, síndrome de hiper-reflexia e insuficiência renal.

De acordo com as normas do Departamento de Obstetrícia, definiu-se: mortalidade perinatal como o óbito ocorrido entre a $20^{\text {a }}$ semana de gestação até 28 dias após o nascimento; sofrimento fetal agudo como a condição detectada por alterações na cardiotocografia intraparto, tais como redução da variabilidade dos movimentos cardíacos fetais, bradicardia sustentada, desacelerações tipo DIP II e umbilicais profundas. Criança pequena para idade gestacional foi definida como aquela com peso abaixo do percentil 10 da curva de Lubchenco ${ }^{23}$. Hipoglicemia foi definida como nível de glicose sérica inferior a $40 \mathrm{mg} / \mathrm{dl}$; policitemia, como hematócrito venoso superiora a $75 \%$ e anemia como hematócrito inferior a $40 \%$ na primeira semana de vida ${ }^{24}$.

A interrupção da gestação foi indicada por sofrimento fetal agudo; piora no estado de saúde materno e/ou Doppler de ducto venoso alterado. Todos os dados referentes aos resultados perinatais foram obtidos dos prontuários das pacientes.

Para análise estatística, utilizou-se o programa estatístico SAS 8.2. Inicialmente calculou-se a frequência dos resultados perinatais adversos (RPA) isolados para as relações IPACM/IPAU e IRACM/IRAU, e a frequência dos RPA para as duas relações de centralização alteradas, utilizando-se os testes exato de Fisher ou $\chi^{2}$ de Pearson, considerando-se $\mathrm{p}<0,05$ como estatisticamente significativo.

O cálculo da idade gestacional pelo método de Capurro $^{25}$, peso e estatura, foram comparados entre os grupos de centralização (um índice alterado e os dois índices alterados) através do teste de Mann-Whitney ${ }^{26}$. Como se obteve valor de p para estatura e peso limítrofes, realizou-se regressão logística múltipla para o cálculo de Odds Ratio ajustado para os mesmos (Capurro, peso e estatura) com a finalidade de eliminar o efeito destas nas estimativas de riscos (Odds Ratio). Os resultados perinatais adversos da amostra total de casos de centralização foram avaliados de acordo com a idade gestacional, utilizando-se o teste do $\chi^{2}$ de tendência de Cochrane-Armitage ${ }^{26}$. 
Por se tratar de estudo retrospectivo, não se propõe o termo de consentimento informado, solicitando-se sua dispensa ao Comitê de Ética em Pesquisa. Os dados obtidos foram utilizados de maneira confidencial, obedecendo aos preceitos do Código de Ética Médica para utilização científica de dados de pacientes e respeitados as normas regulamentadoras para pesquisas envolvendo seres humanos.

\section{Resultados}

Foram avaliados 151 casos de centralização de fluxo sanguíneo fetal, diagnosticados pelas relações IPACM/IPAU e/ou IRACM/IRAU alterados. A relação IRACM/IRAU apresentou-se alterada em 94,7\% dos casos e a relação IPACM/ APAU mostrou-se alterada em 93\%. Da amostra total, em $76,4 \%$ o diagnóstico de centralização de fluxo sanguíneo ocorreu em idade gestacional inferior a $35^{a}$ semana.

O tempo entre o diagnóstico ultrassonográfico de centralização de fluxo sanguíneo fetal e o nascimento não excedeu 15 dias. Nasceram no mesmo dia do diagnóstico ultrassonográfico $23,5 \%$ e, até sete dias após, $62,2 \%$ dos fetos.

A idade do concepto avaliada pelo teste de Capurro variou de 26 a 42 semanas, com média de 34,6. O peso dos recém-nascidos variou de 360 a $4.930 \mathrm{~g}$, com média de $1.804 \mathrm{~g}$, e a estatura variou de 26 a $55 \mathrm{~cm}$, com média de $40,7 \mathrm{~cm}$.
Neste estudo ocorreram 25 óbitos perinatais $(16,5 \%)$, sendo 12 fetais. Os óbitos fetais ocorreram entre a $23^{\mathrm{a}} \mathrm{e}$ $32^{\mathrm{a}}$ semanas, e os ocorridos após o parto apresentaram, ao nascimento, idade gestacional entre 25 e 30 semanas. A presença de componente diastólico ausente ou reverso ocorreu em 21 casos, com evolução para óbito fetal em quatro casos, e para óbito pós-natal em seis casos. A paciente com diástole reversa na artéria umbilical apresentava como doença de base pré-eclâmpsia, evoluindo para parto cesárea por sofrimento fetal agudo com 32 semanas (peso do recém nato: $920 \mathrm{~g}$ ).

A análise isolada da associação entre os resultados perinatais e o IPACM/IPAU normal e alterado, assim como para o IRACM/IRAU normal e alterado, não foi significativa. A análise entre um ou outro índice alterado e os dois índices cérebro-placentários alterados não foi significativa para resultados perinatais adversos (Tabela 1). A análise, utilizando-se Odds Ratio ajustada pela idade gestacional, revelou que quando os dois índices para diagnóstico de centralização apresentaram-se alterados ocorreu 4,2 vezes mais anemia, 1,6 vezes mais policitemia e 2,4 vezes mais crianças com Apgar inferior a sete no quinto minuto.

Analisando-se os resultados perinatais adversos para o grupo em que os dois índices apresentaram-se alterados (Tabela 1 ), foi constatado que $62,5 \%$ dos recém-natos necessitaram de internação em UTI, com média de internação

Tabela 1 - Resultados perinatais adversos (mais significativos) para um ou outro índice alterado e para ambos alterados

\begin{tabular}{|c|c|c|c|c|c|c|c|c|}
\hline \multirow[b]{2}{*}{ Resultados perinatais adversos } & \multicolumn{2}{|c|}{ Um índice alterado } & \multicolumn{2}{|c|}{ Ambos os índices alterados } & \multirow[b]{2}{*}{ Valor de p } & \multicolumn{3}{|c|}{ OR ajustado (IC 95\%) } \\
\hline & n & $\%$ & n & $\%$ & & OR & $\begin{array}{l}\text { Limite } \\
\text { inferior }\end{array}$ & $\begin{array}{l}\text { Limite } \\
\text { superior }\end{array}$ \\
\hline Mortalidade perinatal & & & & & $0,8^{\circ}$ & & & \\
\hline $\operatorname{Sim}$ & 8 & 17,4 & 17 & 12,2 & & 0,6 & 0,1 & 3,3 \\
\hline Septicemia & & & & & $0,7^{\circ}$ & & & \\
\hline Pequeno para idade gestacional & & & & & $0,1^{\circ}$ & & & \\
\hline Não & 17 & 37 & 26 & 24,8 & & & & \\
\hline $\operatorname{Sim}$ & 29 & 63 & 79 & 75,2 & & 1,0 & 0,2 & 3,4 \\
\hline Sofrimento fetal agudo & & & & & $0,6^{\circ}$ & & & \\
\hline Não & 26 & 60,5 & 66 & 64,7 & & & & \\
\hline Sim & - & - & 4 & 4,2 & & & & \\
\hline Membrana hialina & & & & & $0,4^{0}$ & & & \\
\hline Não & 37 & 88,1 & 80 & 83,3 & & & & \\
\hline Sim & 5 & 11,9 & 16 & 16,7 & & 0,9 & 0,2 & 3,5 \\
\hline Anemia & & & & & $0,1^{b}$ & & & \\
\hline Não & 41 & 97,6 & 84 & 87,5 & & & & \\
\hline Sim & 1 & 2,4 & 12 & 12,5 & & 4,2 & 0,4 & 36,6 \\
\hline
\end{tabular}

${ }^{a}$ teste do $\chi^{2} ;{ }^{b}$ teste exato de Fisher; * um caso em que a criança morreu uma hora após o nascimento foi excluída. 
de 24 dias. $\mathrm{O}$ índice de Apgar menor que sete no quinto minuto ocorreu em 9,5\% dos casos; conceptos pequenos para a idade gestacional (PIG) em $75,2 \%$, sofrimento fetal agudo em $35,3 \%$, hipoglicemia em $84,4 \%$, policitemia em $8,3 \%$, enterocolite necrosante em $4,2 \%$; hemorragia cerebral em $2,1 \%$, insuficiência renal em $2,1 \%$, doença de membrana hialina em $16,7 \%$, hiper-reflexia em $3,1 \%$, anemia em $12,5 \%$ e síndromes convulsivas em $3 \%$. Hemorragia pulmonar ocorreu em apenas um caso, no grupo em que um ou outro índice cérebro-placentário mostrou-se alterado.

Analisando-se os resultados perinatais adversos da amostra total de casos com centralização de fluxo sanguíneo, de acordo com a idade gestacional, constatou-se associação significativa com internação em UTI neonatal, fetos pequenos para a idade gestacional, septicemia, enterocolite necrosante, insuficiência renal, doença de membrana hialina e anemia (Tabela 2). Não houve associação significativa com sofrimento fetal agudo, hipoglicemia, policitemia, hemorragia cerebral, hiper reflexia e síndromes convulsivas. $\mathrm{O}$ teste de tendência demonstra associação negativa no decorrer da idade gestacional, ou seja, os resultados perinatais adversos foram menos frequentes com o avanço da idade gestacional (Tabela 2).

A necessidade de UTI foi proporcionalmente maior quanto menor a idade gestacional. Em idade gestacional superior a $35^{\mathrm{a}}$ semana, $6,9 \%$ dos recém-natos necessitou de internação. Os fetos pequenos para a idade gestacional apresentaram comportamento semelhante, com incidência de $12 \%$ em idade superior a $35^{\mathrm{a}}$ semana. A maior incidência de sofrimento fetal agudo ocorreu na faixa entre a $30^{\mathrm{a}}$ e $32^{\mathrm{a}}$ semanas, sendo de $38,5 \%$. A grande maioria, ou seja, $80,9 \%$ dos casos, ocorreu com mais de 35 semanas. Um dos fetos com centralização de fluxo sanguíneo (IPACAM/IPAU =0,39 e diástole zero na artéria umbilical) apresentou hemorragia cerebral, enterocolite necrosante e insuficiência renal. A criança pesou $870 \mathrm{~g}$ com Apgar no quinto minuto de um, e Capurro de 32 semanas. A

Tabela 2 - Resultados perinatais adversos, da amostra total de fetos com centralização de fluxo sanguíneo, de acordo com a idade gestacional

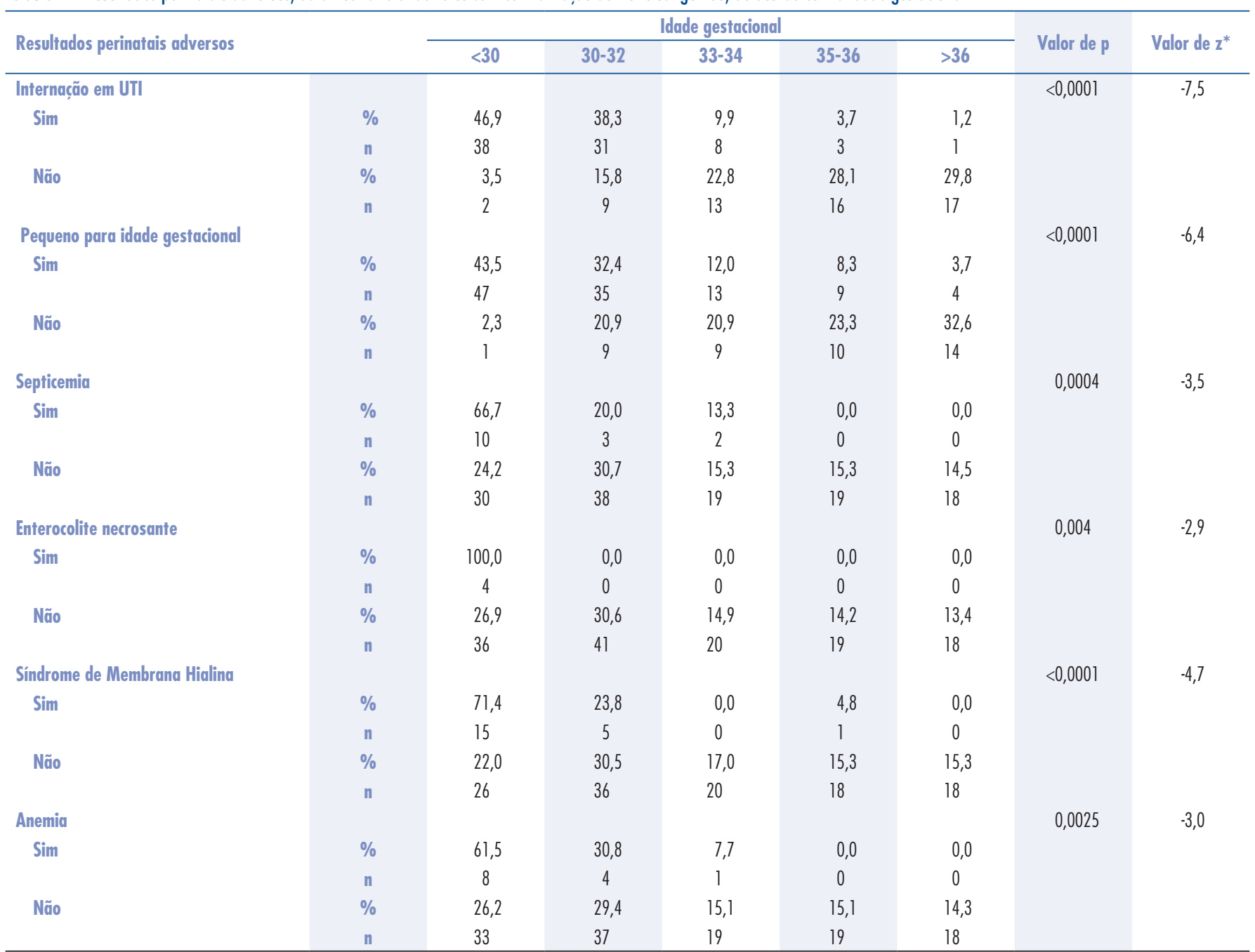

* Teste de tendência de Cochrane-Armitage. 
placenta apresentou $10 \%$ de área infartada e a patologia materna era pré-eclâmpsia.

Quatro crianças apresentaram enterocolite necrosante, com Capurro variando de 29 a 32 semanas, peso variando de 870 a $1.400 \mathrm{~g}$, e relação S/D da artéria umbilical variando de 5 a 6,5 , sendo um caso com diástole zero.

Hemorragia pulmonar ocorreu em apenas um caso, com IPACM/IPAU =1,1 e IRACM/IRAU =0,97 e relação $\mathrm{S} / \mathrm{D}$ da artéria umbilical=8,2. A gestante não apresentava patologia de base. A criança pesou $650 \mathrm{~g}$, com Capurro de 27 semanas. Síndromes convulsivas ocorreram em cinco casos, com peso variando de 650 a 1.680 g e Capurro variando de 30 a 34 semanas. Um caso apresentou diástole zero na artéria umbilical, e nos outros a relação S/D da artéria umbilical variou de 3,6 a 6,8. Infartos placentários foram diagnosticados em 40 casos, mas nem todas as placentas deste estudo foram submetidas ao exame histológico.

\section{Discussão}

$\mathrm{Na}$ clínica obstétrica um método eficiente de rastreamento de sofrimento fetal é de fundamental importância, não apenas para redução da mortalidade, mas para garantir qualidade de vida futura.

De uma forma geral, os fetos que evoluem para centralização de fluxo sanguíneo apresentam associação com prematuridade e baixo peso ${ }^{12,14,27,28}$, fatos que podem ser constatados pelos resultados deste estudo, em que a média da idade gestacional calculada pelo método de Capurro foi de 34,6 semanas, o peso fetal médio de $1.804 \mathrm{~g}$. A média de peso ao nascimento em um estudo na Sérvia foi de $1.327 \mathrm{~g}^{28}$ e, no Kuwait, de 1.835 g, com idade gestacional média de 35,4 semanas ${ }^{12}$.

A análise isolada da associação entre os resultados perinatais e o IPACM/IPAU normal e alterado, assim como para o IRACM/IRAU normal e alterado não foi significativa, provavelmente porque quando um índice estava alterado, o outro se encontrava normal, mas com valores muito próximos do valor considerado alterado. $\mathrm{O}$ índice de resistência apresenta desvantagem em relação ao índice de pulsatilidade na presença de componente diastólico ausente na artéria umbilical, pois nesses casos o índice de resistência é igual à unidade, impossibilitando a análise numérica da relação cérebro-placentária.

A análise comparativa entre as relações doplervelocimétricas alteradas realizadas com o índice de pulsatilidade e com o índice de resistência não demonstrou diferenças significativas para resultados perinatais adversos. O mesmo ocorreu quando os dois índices se mostraram alterados.

A morbidade dos fetos com centralização de fluxo sanguíneo fetal na literatura é considerável, em torno de $93 \%{ }^{28}$. Neste estudo, a ocorrência de crianças pequenas para a idade gestacional foi de $75,2 \%$, sofrimento fetal agudo em 35,3\% e hipoglicemia em 84,4\% dos casos, quando os dois índices se mostraram alterados. Constatouse também alta incidência de internação em UTI neonatal por períodos prolongados (com média de internação de 24 dias), fato constatado em outros estudos ${ }^{12,13}$.

O índice de Apgar menor que sete no quinto minuto ocorreu em 9,5\% dos casos. Outros estudos demonstram que o índice de Apgar não foi significativo para resultados perinatais adversos ${ }^{12,15}$. Alguns pesquisadores ignoram o índice de Apgar no quinto minuto por considerá-lo inadequado ao diagnóstico de hipóxia neonatal, pois somente $15 \%$ dos recém-nascidos afetados por paralisia cerebral apresentam índices de Apgar baixos ${ }^{29}$. A incidência de hipoglicemia foi alta, quando os dois índices se apresentaram alterados, e mesmo quando um ou outro se mostrou alterado. Neste estudo, apesar do grande número de prematuros, a incidência de doença de membrana hialina se mostrou relativamente baixa, provavelmente em decorrência de hipoxemia crônica e pelo uso de corticoterapia profilática.

A concomitância de centralização de fluxo com componente diastólico ausente ou reverso na artéria umbilical tem sido associada com maior mortalidade ${ }^{12,30,31}$. Nete estudo houve 25 óbitos perinatais $(16,5 \%)$, sendo que 10 apresentaram componente diastólico ausente ou reverso na artéria umbilical. A mortalidade perinatal relatada em um estudo na Sérvia foi de 14,3\%. A presença de componente diastólico ausente ou reverso na artéria umbilical tem sido associada com maior incidência de enterocoline necrosante, cuja intercorrência apresenta alta mortalidade, em torno de $50 \%{ }^{31}$. Notou-se aumento da mortalidade e morbidade em fetos com centralização de fluxo em gestações com menos de 35 semanas. Todos os óbitos fetais ocorreram com idade gestacional entre 25 e 30 semanas.

Alguns pesquisadores têm afirmado que a relação entre os índices cerebrais e placentários prediz problemas perinatais somente em fetos com menos de 34 semanas de gestação. Uma explicação para essa ocorrência seria sobrecarga do feto prematuro na tentativa de compensação da hipóxia cerebral ${ }^{32}$. O mecanismo de controle do fluxo sanguíneo fetal seria incompleto em neonatos prematuros, e a asfixia poderia contribuir para a deficiência no mecanismo de autorregulação nessas crianças ${ }^{32,33}$. Entretanto, alguns pesquisadores admitem que a doplervelocimetria da artéria cerebral pode ser utilizada na avaliação da vitalidade fetal no final do terceiro trimestre, e que a redistribuição sanguínea fetal poderia ocorrer até mesmo na presença de doplervelocimetria normal na artéria umbilical ${ }^{34}$.

O conhecimento dos fatores de ajuste circulatório e seu mecanismo de ação não são bem conhecidos. Provavelmente, quando a pressão parcial de $\mathrm{O}_{2}$ diminui 
e a de $\mathrm{CO}_{2}$ se eleva acima de um determinado nível, os quimiorreceptores aórticos e carotídeos podem ser ativados, regulando a resposta vasodilatadora central com a finalidade de garantir oxigenação adequada ao cérebro fetal ${ }^{35}$. A centralização de fluxo sanguíneo fetal parece ser um mecanismo benigno de adaptação que contribui para a prevenção de lesões cerebrais severas. Os danos neurológicos nas crianças que apresentaram distribuição preferencial de sangue para o cérebro são contraditórios ${ }^{36-}$ ${ }^{40}$. Estudos recentes demonstraram que o mecanismo de redistribuição sanguínea não protege a criança de todos os problemas neurológicos, sendo frequentes as alterações comportamentais e emocionais na infância ${ }^{39}$. Parece que a centralização de fluxo sanguíneo protege preferencialmente a região frontal do cérebro, que está envolvida na área emocional, cognitiva e nos processos motivacionais ${ }^{40}$.

O peso do feto extremamente baixo aumenta o risco de mortalidade e morbidade perinatal, mas a variável mais significativa que pode estar associada ao desenvolvimento neurológico da criança é a idade gestacional ${ }^{41}$. Neste estudo, os resultados perinatais adversos foram menores com o aumento progressivo da idade gestacional.
A centralização de fluxo sanguíneo fetal ocorre como um mecanismo de adaptação à hipoxemia crônica. Entretanto, em gestação normal, com mais de 34 semanas, o fluxo nas artérias cerebrais apresenta baixa resistência, e as relações cérebro-placentárias podem ser maiores que $u^{42}$. Recomenda-se uma análise criteriosa de sua presença como instrumento de uso para decisões obstétricas. A insuficiência placentária é uma condição progressiva, cujo tratamento é a resolução da gestação. $\mathrm{O}$ momento ideal para se definir esa conduta deve levar em consideração os danos causados pela permanência intraútero sob regime de hipóxia e as complicações decorrentes da prematuridade ${ }^{43}$.

Constatou-se, neste estudo, uma associação significativa das relações IPACM/IPAU e IRACM/IRAU, no decorrer da idade gestacional, para necessidade de cuidados intensivos neonatais, fetos pequenos para a idade gestacional, septicemia, enterocolite necrosante, insuficiência renal, doença de membrana hialina e anemia fetal. Não houve diferença significativa entre um ou outro índice alterado (IPACM/IPAU e IRACM/IRAU) para resultados perinatais adversos.

\section{Referências}

1. Alfirevic Z, Neilson JP. Doppler ultrasonography in high-risk pregnancies: systematic review with meta-analysis. Am J Obstet Gynecol. 1995; 172(5):1379-87.

2. McCowan LM, Harding JE, Roberts AB, Barker SE, Ford C, Stewart AW. A pilot randomized controlled trial of two regiments of fetal surveillance for small-for-gestational-age fetuses with normal results of umbilical artery Doppler velocimetry. Am J Obstet Gynecol. 2000; 182(1 Pt 1):81-6.

3. Westergaard HB, Langhoff-Ross J, Lingman G, Marsál K, Kreiner S. A critical appraisal of the use of umbilical artery Doppler ultrasound in high-risk pregnancies: use of meta-analyses in evidence-based obstetrics. Ultrasound Obstet Gynecol. $2001 ; 17(6): 466-76$.

4. Wladimiroff JW, Tonge HM, Stewart PA. Doppler ultrasound assessment of cerebral blood flow in the human fetus. $\mathrm{Br} J$ Obstet Gynaecol. 1986;93(5):471-5.

5. Mari G, Deter RL. Middle cerebral artery flow velocity waveforms in normal and small-for-gestational-age fetuses. J Obstet Gynecol. 1992; 166(4): 1262-70.

6. Arbeille PH, Roncin A, Berson M, Patat F, Pourcelot L. Exploration of the fetal cerebral blood flow by duplex Doppler - linear array system in normal and pathological pregnancies. Ultrasound Med Biol. 1987; 13(6):329-37.

7. Peeters LLH, Sheldon RE, Jones MD Jr, Makowski EL, Meschia G. Blood flow to fetal organs as a function of arterial oxygen content. Am J Obstet Gynaecol. 1979;135(5):637-46.
8. Vyas S, Nicolaides $\mathrm{KH}$, Bower S, Campbell S. Middle cerebral artery flow velocity waveforms in fetal hypoxaemia. Br J Obstet Gynaecol. 1990;97(9):797-803.

9. Arduini D, Rizzo G. Prediction of fetal outcome in small for gestacional age fetuses: comparison of Doppler measurements obtained from different fetal vessels. J Perinat Med. 1992;20(1):29-38.

10. Gramellini F, Folli MC, Raboni S, Vadora E, Medialdi A. Cerebralumbilical Doppler ratio as a predictor of adverse perinatal outcome. Obstet Gynecol. 1992;79(3):416-20.

11. Arias F. Accuracy of the middle-cerebral-to-umbilical-artery resistance index ratio in the prediction of neonatal outcome in patients at high risk for fetal and neonatal complications. Am J Obstet Gynecol. 1994; 171(6):1541-5.

12. Makhseed M, Jirous J, Ahmed MA, Viswanathan DL. Middle cerebral artery to umbilical artery resistance index ratio in the prediction of neonatal outcome. Int J Gynaecol Obstet. 2000;71 (2):1 19-25.

13. Ebrashy A, Azmy O, Ibrahim M, Waly M, Edris A. Middle cerebral/ umbilical artery resistance index ratio as sensitive parameter for fetal well-being and neonatal outcome in patients with preeclampsia: case-control study. Croat Med J. 2005;46(5):821-5.

14. Yalti S, Oral O, Gürbüz B, Özden S, Atar F. Ratio of middle cerebral to umbilical artery blood velocity in preeclamptic \& hypertensive women in the prediction of poor perinatal outcome. Indian J Med Res. 2004;120(1):44-50.

15. Borowski D, Czuba B, Włoch A, Wyrwas D, Hincz P, Szaflik K, et al. Doppler assessment of the fetal asphyxia in pregnancies 
complicated by gestational hypertension and intrauterine growth retardation. Ginekol Pol. 2006;77(3): 184-9.

16. Cheema R, Dubiel M, Gudmundsson S. Fetal brain sparing is strongly related to the degree of increased placental vascular impedance. J Perinat Med. 2006;34(4):3 18-22.

17. Teixeira JM, Glover V, Fisk NM. Acute cerebral redistribution in response to invasive procedures in the human fetus. Am J Obstet Gynecol. 1999;181(4):1018-25.

18. Donofrio MT, Bremer YA, Schieken RM, Gennings C, Morton LD, Eidem BW, et al. Autoregulation of cerebral blood flow in fetuses with congenital heart disease: the brain sparing effect. Pediatr Cardiol. 2003;24(5):436-43.

19. Gosling RG, King DH. Arterial assessment by Doppler-shift ultrasound. Proc R Soc Med. 1974;67(6 Pt 1):447-9.

20. Pourcelot L. Applications cliniques de l'examen Doppler transcutané. In: Peronneau $P$, éditeur. Vélocimetrie ultrasonore Doppler [Les Colloques de I'Institut National de la Santé et de la Recherche Médicale, 34]. Paris: Inserm; 1974. p. 213-40.

21. [No authors listed]. Recommendations on the use of ultrasound and Doppler technology in clinical obstetrics and gynecology. FIGO Study Group on the Assessment of New Technology. Int J Gynaecol Obstet. 1992;37(3):221-7.

22. Arduini $D$, Rizzo G. Normal values of pulsatility index from fetal vessels: a cross-sectional study on 1556 healthy fetuses. J Perinat Med. 1990;18(3):165-72.

23. Lubchenco LO, Hansman C, Dressler M, Boyd E. Intrauterine growth as estimated from liveborn birth-weight data at 24 to 42 weeks of gestation. Pediatrics. 1963;32:793-800.

24. Marba STM, Mezzacappa Filho F. Manual de neonatologia da Unicamp. 2a ed. Rio de Janeiro: Revinter; 2009.

25. Capurro H, Konichezky S, Fonseca D, Caldeyro-Barcia R. A simplified method for diagnosis of gestational age in the newborn infant. J Pediatr. 1978;93(1):120-2.

26. Altman DG. Pratical statistics for medical research. London: Chapman \& Hall; 1991.

27. Sterne G, Shields LE, Dubinsky TJ. Abnormal fetal cerebral and umbilical Doppler measurements in fetuses with intrauterine growth restriction predicts the severity of perinatal morbidity. J Clin Ultrasound. $2001 ; 29(3): 146-51$.

28. Miković Z, Mandić V, Djukić M, Egić A, Filimonović D, Cerović N, et al. Longitudinal analysis of arterial Doppler parameters in growth retarded fetuses. Srp Arh Celok Lek. 2003;131(1-2):21-5.

29. Freeman JM, Nelson KB. Intrapartum asphyxia and cerebral palsy. Pediatrics. 1988;82(2):240-9.

30. Ferrazzi E, Bozzo M, Rigano S, Bellotti M, Morabito A, Pardi G, et al. Temporal sequence of abnormal Doppler changes in the peripheral and central circulatory systems of the severely growthrestricted fetus. Ultrasound Obstet Gynecol. 2002;19(2):140-6.

31. Bhatt AB, Tank PD, Barmade KB, Damania KR. Abnormal Doppler flow velocimetry in the growth restricted foetus as a predictor for necrotising enterocolitis. J Postgrad Med. 2002;48(3): 182-5.

32. Bahado-Singh RO, Kovanci E, Jeffres A, Oz U, Deren O, Copel J, et al. The Doppler cerebroplacental ratio and perinatal outcome in intrauterine growth restriction. Am J Obstet Gynecol. 1999; 180(3 Pt 1):750-6.

33. Milligan DW. Failure of autoregulation and intraventricular haemorrhage in preterm infants. Lancet. 1980; 1 (8174):896-8.

34. Hershkovitz R, Kingdom JC, Geary M, Rodeck CH. Fetal cerebral blood flow redistribution in late gestation: identification of compromise in small fetuses with normal umbilical artery Doppler. Ultrasound Obstet Gynecol. 2000; 15(3):209-12.

35. Dawes GS, Lewis BV, Milligan JE, Roach MR, Talner NS. Vasomotor responses in the hind limbs of foetal and new-born lambs to asphyxia and aortic chemoreceptor stimulation. J Physiol. 1968; 195(1):55-81.

36. Scherjon SA, Oosting $H$, Smolders-DeHaas $H$, Zondervan HA, Kok $\mathrm{JH}$. Neurodevelopmental outcome at three years of age after fetal 'brain-sparing'. Early Hum Dev. 1998;52(1):67-79.

37. Scherjon S, Briët J, Oosting H, Kok J. The discrepancy between maturation of visual-evoked potentials and cognitive outcome at five years in very preterm infants with and without hemodynamic signs of fetal brain-sparing. Pediatrics. 2000;105(2):385-91.

38. Kok JH, Prick L, Merckel E, Everhard Y, Verkerk GJ, Scherjon SA. Visual function at 11 years of age in preterm-born children with and without fetal brain sparing. Pediatrics. 2007; $119(6)$ : e 1342-50.

39. Roza SJ, Steegers EA, Verburg BO, Jaddoe VW, Moll HA, Hofman $A$, et al. What is spared by fetal brain-sparing? Fetal circulatory redistribution and behavioral problems in the general population. Am J Epidemiol. 2008;168(10): 1 145-52.

40. Dubiel M, Gunnarsson GO, Gudmundsson S. Blood redistribution in the fetal brain during chronic hypoxia. Ultrasound Obstet Gynecol. 2002;20(2):117-21.

41. Valcamonico A, Accorsi P, Sanzeni C, Martelli P, La Boria P, Cavazza A, et al. Mid- and long-term outcome of extremely low birth weight (ELBW) infants: an analysis of prognostic factors. J Matern Fetal Neonatal Med. 2007;20(6):465-71.

42. Botsis D, Vrachnis N, Christodoulakos G. Doppler assessment of the intrauterine growth-restricted fetus. Ann N Y Acad Sci. 2006; 1092:297-303

43. Villas-Bôas JMS, Maestá I, Consonni M. Mecanismo de centralização: da insuficiência placentária à adaptação circulatória fetal. Rev Bras Ginecol Obstet. 2008;30(7):366-71. 